\title{
Clinical significance of mechanical ventilation on ischemic-reperfusion injury caused by lung chest trauma and VEGF expression levels in peripheral blood
}

\author{
ZHONG-YI SUN* ${ }^{*}$ HONG-GANG XIA* , DE-QING ZHU* , LI-MIN DENG, PENG-ZHI ZHU and DONG-BIN WANG \\ Department of Cardiothoracic Surgery, Tianjin Hospital, Tianjin 300210, P.R. China
}

Received September 21, 2016; Accepted February 9, 2017

DOI: $10.3892 /$ etm.2017.4825

\begin{abstract}
We investigated the clinical significance of mechanical ventilation on ischemic-reperfusion injury caused by lung chest trauma as well as vascular endothelial growth factor (VEGF) expression levels in peripheral blood. Sixty-eight patients with severe chest trauma complicated with acute respiratory distress syndrome that were treated at our Tianjin Hospital from September 2013 to July 2016 were recruited. These patients were randomly and evenly divided into two groups, the research group and the control group. Thirty-four age and gender matched healthy people were selected as the normal group. Routine treatment was given to both the research and control groups, but mechanical ventilation was used in the research group. We detected pulmonary vascular resistance (PVR) and alveolar-arterial oxygen difference $\left(\mathrm{AaDO}_{2}\right)$ for patients in both groups before treatment, and after treatment for 1, 3, 6 and $12 \mathrm{~h}$. We also tested PMN, superoxide dismutase (SOD), malondialdehyde (MDA), $\mathrm{NO}$ and Ang II value $30 \mathrm{~min}$ before and after treatment. We used the ELISA-test to detect VEGF expression levels in peripheral blood, followed by a statistical analysis. PVR levels of different time points in the research group were significantly lower than control group after treatment. The $\mathrm{AaDO}_{2}$ value of the control group is much smaller than research group $(\mathrm{P}<0.5)$ after treatment for 1,3 or $6 \mathrm{~h}$. PMN count difference and MDA level in the research group is significantly lower than the control group after treatment for $30 \mathrm{~min}$, but SOD and NO levels are much higher. Ang II levels of the research group in left atrial blood is significantly lower than control group $(\mathrm{P}<0.05)$. By comparing the hospitalization times, we found that patients in the research group have a shorter duration in hospital than the control group; differences are statistically
\end{abstract}

Correspondence to: Dr Dong-Bin Wang, Department of Cardiothoracic Surgery, Tianjin Hospital, 406 South Jiefang Road, Tianjin 300210, P.R. China

E-mail:wf8i16@163.com

${ }^{*}$ Contributed equally

Key words: chest trauma, mechanical ventilation, vascular endothelial growth factor, lung ischemic-reperfusion injury significant $(\mathrm{P}<0.05)$. Additionally, compared to control group, research group VEGF expression levels in peripheral blood are significantly lower $(\mathrm{P}<0.05)$. Therefore, mechanical ventilation can reduce the high VEGF expression levels in serum caused by ischemic-reperfusion and can be used for clinical application.

\section{Introduction}

Severe chest trauma is a disease with acute onset, rapid development and is often associated with acute respiratory distress syndrome (ARDS). The mortality of this disease is extremely high without a timely treatment, and can be between 50 and $70 \%$ (1). Therefore, an effective treatment for severe chest trauma patients can not only improve prognosis to enhance survival, but also reduce the occurrence of chain reaction after severe chest injury $(2,3)$.

A causality has been found between lung ischemic-reperfusion injury and inflammatory response in various complications caused by chest trauma. Some severe cases can develop into respiratory distress syndrome, which will affect the patients' perioperative life seriously. Previous studies have found that lung ischemic preconditioning can significantly improve postoperative $\mathrm{PaO}_{2}$ levels and remarkably reduce pulmonary artery pressure, lung wet/dry ratio and malondialdehyde levels, which may to some extent prevent the damage of lung tissue (4).

Previous studies report that ischemia-reperfusion injury can cause an increase in levels of blood neutrophils (PMN), peripheral blood superoxide dismutase and malondialdehyde (SOD and MDA), NO and Ang II, which probably related to ischemia, hypoxia and the stress of hypoxia of the lung (5-7). Additionally, vascular endothelial growth factor (VEGF) can induce various physiological and pathological processes. Its expression levels can be improved markedly in angiogenesis, vascular proliferation, tissue ischemia and hypoxia conditions to meet the needs of aerobic metabolism for injury tissue, which plays an important role in tissue reconstruction. Therefore, some scholars consider that VEGF expression levels can be monitored as one of the serological index of lung ischemia-reperfusion (8). On the basis of previous studies, our study investigated the effects of mechanical ventilation on ischemic-reperfusion injury caused by lung chest trauma and VEGF expression levels in peripheral blood. 
Table I. Clinical settings of investigated patients.

\begin{tabular}{lccccccc}
\hline Groups & Cases & $\begin{array}{c}\text { Gender } \\
\text { (male/female) }\end{array}$ & Age (years) & BMI $\left(\mathrm{kg} / \mathrm{m}^{2}\right)$ & $\begin{array}{c}\text { NYHA } \\
(\mathrm{II} / \mathrm{III})\end{array}$ & $\begin{array}{c}\text { Bleeding } \\
\text { volume }\end{array}$ & $\begin{array}{c}\text { Treatment } \\
\text { time (min) }\end{array}$ \\
\hline RG & 34 & $19 / 16$ & $52.4 \pm 12.3$ & $23.3 \pm 1.9$ & $29 / 31$ & $172.3 \pm 12.6$ & $51.2 \pm 12.7$ \\
CG & 34 & $20 / 14$ & $55.6 \pm 7.2$ & $22.4 \pm 2.8$ & $28 / 32$ & $187.8 \pm 28.5$ & $53.2 \pm 22.1$ \\
F/ $\chi^{2}$ value & - & 0.34 & 0.27 & 0.62 & 0.33 & 0.48 & 0.99 \\
P-value & - & 0.82 & 0.37 & 0.44 & 0.68 & 0.55 & 0.17 \\
\hline
\end{tabular}

RG, research group; CG, control group.

\section{Materials and methods}

Sample selection. Sixty-eight patients with severe chest trauma complicated with ARDS who were treated at our hospital from September 2013 to July 2016 were recruited. These patients were randomly and evenly divided into two groups, the research group and the control group. The control group included 19 males and 15 females, aged 19-72 years, average $45.3 \pm 12.5$ years, 14 injury cases resulting from traffic accidents, 7 cases of fall, 6 cases of crush injury, 5 bluntly attack injury cases and other 2 cases; 7 cases were single chest trauma and 27 cases were multiple or compound injury. The research group included 18 males and 16 females, aged 20-64 years (44 \pm 2.51$) ; 16$ injury cases due to traffic accidents, 6 fall injury cases, 7 crush injury cases, 4 bluntly attack injury cases and 1 other case; 8 cases were single chest trauma, and 26 cases were multiple or compound injury. The 34 cases in the control group were treated in Tianjin Hospital's Physical and Examination Center simultaneously. No significant differences of clinical data were found between these two groups $(\mathrm{P}>0.05)$, and they are comparable. This study was approved by the Medical Ethics Committee of Tianjin Hospital and written informed consent was obtained from the patients.

Inclusion and exclusion criteria. Inclusion criteria: i) NYHA cardiac function class II-III; and ii) diagnosis of chest trauma in patients by clinical symptoms, signs, medical history, laboratories and control laboratories.

Exclusion criteria: i) patients with comprehensive symptoms of pneumothorax, severe arrhythmia and myocardial; ii) patients with cardiac pulmonary edema caused by respiratory distress and patients with non-completed clinical data; and iii) patients with mental illness or disturbance of consciousness.

Diagnostic criteria. All patients chosen in our study have significant traumatic injuries and acute onset. The clinical symptoms are faster respiratory rates (RR, $>28$ times/min), and pulmonary artery wedge pressure (PAWP, $\leq 18 \mathrm{mmHg}$ ), the symptoms still not being able to ease after a general oxygen therapy, and a cloudy shadow was found in patients' lung by using physical and chemical inspection and X-ray examination.

Methods. We closely monitored the patient vital signs and established a venous channel after they were hospitalized. We administered routine treatment to these two groups of patients according to parameters such as oxygen uptake, respiratory stimulant, anti-infection, bronchiectasis, correct water electrolyte and acid-base imbalance, and nutritional support. Apart from the routine treatment, mechanical ventilation was used in the research group. The BIPAP ventilator (manufactured by Philips Respironics, Inc., Murrysville, PA, USA) was used to help patients breathe, set up S/T model for 16 times/min respiratory rate, $8 \mathrm{~cm} / \mathrm{H}_{2} \mathrm{O}$ oxygen pressure, $4 \mathrm{~cm} / \mathrm{H}_{2} \mathrm{O}$ EPAP, and $35-60 \%$ oxygen concentration.

Observation targets. We observed the research and control group blood gas analysis before treatment and after treatment including $\mathrm{PaCO}_{2}, \mathrm{PaO}_{2}$ and $\mathrm{SaO}_{2}$. We monitored patient lung function after treatment including MVV, FVC, and FEV0.3.

ELISA method to detect VEGF in peripheral blood. Peripheral venous blood was centrifuged for $5 \mathrm{~min}$, and ELISA detection kits (Nanjing Jiancheng Technology Co., Ltd., Nanjing, China) were used to detect VEGF, PMN, SOD, MDA, Ang II and NO levels in peripheral blood. Refer to standardized specifications in the detection kits for test methods and procedures.

SOD, MDA, NO, Ang II level test. SOD is detected by xanthine oxidase, and MDA levels are detected by thiobarbituric acid method; nitric acid reductase method is used to detect NO levels. Ang II is detected by radioimmunoassay.

Statistical analysis. SPSS 19.0 (SPSS, Inc., Chicago, IL, USA) is used for statistical analysis. ANOVA, t-test and $\chi^{2}$ test were used for qualitative data analysis, and Fisher's exact to analyze a fourfold table for patients who do not meet the criteria. In order to compare the qualitative data, the ANOVA test is used and $\mathrm{P}<0.05$ was considered to indicate a statistically significant difference.

\section{Results}

Clinical settings of investigated patients. Statistical analysis of clinical settings for 68 chest trauma patients (Table I) indicated that there were no significant $(\mathrm{P}>0.05)$ statistical differences in gender ratio, average age, hospitalization time and BMI between different groups of patients.

Pulmonary vascular resistance (PVR) comparison. Both the research group (RG) and control group (CG) showed high 
Table II. PVR comparation.

\begin{tabular}{lcccccccc}
\hline & & & \multicolumn{5}{c}{ After treatment } \\
\cline { 4 - 7 } Group & Cases & Before treatment & $1 \mathrm{~h}$ & $3 \mathrm{~h}$ & $6 \mathrm{~h}$ & $12 \mathrm{~h}$ & F-value & P-value \\
\hline RG & 34 & $331.6 \pm 25.7$ & $231.7 \pm 45.3$ & $182.7 \pm 42.8$ & $92.3 \pm 29.6$ & $122.4 \pm 23.6$ & 31.5 & 0.002 \\
CG & 34 & $348.9 \pm 12.6$ & $252.6 \pm 23.8$ & $184.5 \pm 33.6$ & $93.2 \pm 22.8$ & $121.4 \pm 22.5$ & 48.3 & 0.001 \\
F-value & - & 0.33 & 0.41 & 2.57 & 14.7 & 21.2 & - & - \\
P-value & - & 0.37 & 0.33 & 0.02 & 0.01 & 0.01 & - \\
\hline
\end{tabular}

Pulmonary vascular resistance; $\mathrm{RG}$, research group; CG, control group.

Table III. Left atrial ejection SOD, MDA, Ang II and NO level comparation $30 \mathrm{~min}$ before and after treatment.

\begin{tabular}{|c|c|c|c|c|c|c|}
\hline & Group & Cases & Before treatment & After treatment $1 / 2 \mathrm{~h}$ & F-value & P-value \\
\hline \multirow[t]{4}{*}{$\mathrm{SOD}(\mathrm{NU} / \mathrm{ml})$} & RG & 34 & $131.6 \pm 15.7$ & $171.7 \pm 49.3$ & 21.5 & 0.002 \\
\hline & $\mathrm{CG}$ & 34 & $138.9 \pm 12.8$ & $172.6 \pm 22.8$ & 58.3 & 0.001 \\
\hline & T-value & - & 0.33 & 0.41 & - & - \\
\hline & P-value & - & 0.37 & 0.33 & - & - \\
\hline \multirow[t]{4}{*}{$\operatorname{MDA}(\mathrm{nmol} / \mathrm{ml})$} & RG & 34 & $2.28 \pm 0.14$ & $3.38 \pm 1.02$ & 4.37 & 0.017 \\
\hline & $\mathrm{CG}$ & 34 & $2.39 \pm 0.21$ & $3.25 \pm 0.97$ & 5.56 & 0.011 \\
\hline & T-value & - & 0.39 & 12.5 & - & - \\
\hline & P-value & - & 0.66 & 0.01 & - & - \\
\hline \multirow[t]{4}{*}{ Ang II (pg/ml) } & RG & 34 & $77.4 \pm 12.81$ & $168.4 \pm 10.9$ & 2.38 & 0.021 \\
\hline & $\mathrm{CG}$ & 34 & $80.4 \pm 13.9$ & $166.4 \pm 12.8$ & 1.98 & 0.024 \\
\hline & T-value & - & 0.62 & 21.4 & - & - \\
\hline & P-value & - & 0.39 & 0.02 & - & - \\
\hline \multirow[t]{4}{*}{$\mathrm{NO}(\mu \mathrm{mol} / \mathrm{l})$} & RG & 34 & $38.4 \pm 11.3$ & $78.2 \pm 22.4$ & 10.29 & 0.018 \\
\hline & $\mathrm{CG}$ & 34 & $35.7 \pm 14.8$ & $74.4 \pm 23.5$ & 11.33 & 0.014 \\
\hline & T-value & - & 0.26 & 22.8 & - & - \\
\hline & P-value & - & 0.73 & 0.01 & - & - \\
\hline \multirow[t]{4}{*}{ PMN } & RG & 34 & $0.28 \pm 0.56$ & $3.72 \pm 1.15$ & 7.87 & 0.023 \\
\hline & $\mathrm{CG}$ & 34 & $0.35 \pm 0.76$ & $2.41 \pm 1.73$ & 6.59 & 0.032 \\
\hline & T-value & - & 0.38 & 9.94 & - & - \\
\hline & P-value & - & 0.72 & 0.002 & - & - \\
\hline
\end{tabular}

SOD, superoxide dismutase; MDA, malondialdehyde; RG, research group; CG, control group.

PVR levels before treatment but no significant statistical differences $(\mathrm{P}>0.05)$. After treatment, PVR levels in both RG and $\mathrm{CG}$ dropped remarkably at different treatment time points $(\mathrm{P}<0.01)$. There were no significant statistic differences found between these two groups $(\mathrm{P}>0.05)$. Twelve hours after treatment, PVR levels in these two groups gradually stabilized, shown in Table II.

Comparison of peripheral blood PMN, SOD, MDA, Ang II and NO level between $30 \mathrm{~min}$ before and after treatment. As shown in Table III, 30 min after treatment, the neutrophil numbers in peripheral blood was remarkably higher than before treatment; differences were statistically significant $(\mathrm{P}<0.05)$. However, comparison between RG and CG showed that no statistically significant differences were found $(\mathrm{P}>0.05)$. After treatment, the difference in $\mathrm{RG}$ was significantly smaller than $\mathrm{CG}(\mathrm{P}<0.05)$. Thirty minutes after treatment, SOD and NO level in RG were significantly higher but the MDA levels were remarkably lower when compared to those in $\mathrm{CG}$; the difference was statistically significant $(\mathrm{P}<0.05)$. Left atrial ejection (LAE) Ang II levels were also much higher $(\mathrm{P}<0.05)$; no significant difference was found between these two groups $(\mathrm{P}>0.05)$.

Blood gas index and lung function comparison between before treatment and after treatment. Before treatment, there were no significant differences found in $\mathrm{PaCO}_{2}, \mathrm{PaO}_{2}$ and $\mathrm{SaO}_{2}$ levels between the $\mathrm{RG}$ and $\mathrm{CG}$ group ( $\left.\mathrm{P}>0.05\right)$. After treatment, $\mathrm{PaCO}_{2}, \mathrm{PaO}_{2}$ and $\mathrm{SaO}_{2}$ levels in both the RG and $\mathrm{CG}$ group increased and the scale of improvement in RG was 
Table IV. Blood gas index and lung function comparation between before treatment and after treatment.

\begin{tabular}{lcccccccc}
\hline Group & $\mathrm{N}$ & Treatment & $\mathrm{PaCO}_{2}$ & $\mathrm{PaO}_{2}$ & $\mathrm{SaO}_{2}$ & $\mathrm{MVV}$ & $\mathrm{FVC}$ & $\mathrm{FEV0.3}$ \\
\hline $\mathrm{RG}$ & \multirow{2}{*}{34} & Before & $6.96 \pm 0.31$ & $7.21 \pm 0.63$ & $0.83 \pm 0.04$ & $86.52 \pm 18.31$ & $5.44 \pm 1.14$ & $2.59 \pm 0.45$ \\
& & After & $4.38 \pm 0.36$ & $8.94 \pm 1.15$ & $0.99 \pm 0.07$ & $151.36 \pm 36.21^{\mathrm{a}}$ & $5.53 \pm 1.02$ & $3.61 \pm 0.28^{\mathrm{a}}$ \\
T-value & & & 10.93 & 11.24 & 13.25 & 12.82 & 0.49 & 10.89 \\
P-value & & & 0.003 & 0.002 & 0.001 & 0.002 & 0.58 & 0.003 \\
CG & \multirow{2}{*}{34} & Before & $6.91 \pm 0.36$ & $7.25 \pm 0.61$ & $0.84 \pm 0.06$ & $87.32 \pm 29.32$ & $5.61 \pm 0.78$ & $2.67 \pm 0.43$ \\
& & After & $5.94 \pm 0.71$ & $7.35 \pm 1.36$ & $0.88 \pm 0.09$ & $88.64 \pm 28.62$ & $5.51 \pm 1.06$ & $2.54 \pm 0.54$ \\
T-value & & & 0.83 & 0.44 & 0.37 & 0.87 & 0.43 \\
P-value & & & 0.18 & 0.58 & 0.63 & 0.18 & 0.55 \\
\hline
\end{tabular}

Comparing with $\mathrm{CG},{ }^{\mathrm{a}} \mathrm{P}<0.05$. RG, research group; $\mathrm{CG}$, control group.

Table V. Comparation of hospitalized time.

\begin{tabular}{lcc}
\hline Group & Cases & Hospitalized time (days) \\
\hline RG & 34 & $7.87 \pm 1.32$ \\
CG & 34 & $9.36 \pm 2.82$ \\
T-value & - & 10.92 \\
P-value & - & 0.01 \\
\hline
\end{tabular}

RG, research group; CG, control group.

significantly larger than CG; differences were statistically significant $(\mathrm{P}<0.05)$. A significant difference in MVV and FEV0.3 levels were found between RG and CG after treatment; differences were statistically significant $(\mathrm{P}<0.05)$. However, no significant difference in FVC levels was found between two groups ( $\mathrm{P}>0.05)$ (Table IV).

Comparison of hospitalized time. Comparison of hospitalization time between RG and CG found that the RG group is shorter than $\mathrm{CG}$ group; differences were statistically significant $(\mathrm{P}<0.05)$ (Table V).

VEGF expression levels in serum. Comparing VEGF levels in the research group, control group and normal group (NG) it was found that, the RG and CG group showed higher VEGF levels than NG. Peripheral blood VEGF expression levels in
RG was significantly lower than that in $\mathrm{CG}$; differences were statistically significant $(\mathrm{P}<0.05)$ (Table VI).

\section{Discussion}

Rib fractures, pulmonary contusion and hemopneumothorax caused by severe chest trauma can easily affect a patient's respiratory function, or even lead to acute respiratory distress which will aggravate the patient's conditions. Therefore, early diagnosis and treatment are of great importance for the improvement of severe chest trauma and the patient survival. Currently, oxygen therapy is the main treatment to improve the patient respiratory distress and hypoxemia symptoms in clinical practice. However, many clinical studies have shown that mechanical ventilation treatment has a better clinical therapeutic effect for severe chest trauma patients that are accompanied by ARDS (9). Since chest trauma is often accompanied by a massive blood loss and a decrease of lung perfusion, a large amount of oxygen free radicals, leukocytic aggregation and complement activation in ischemia-reperfusion process are considered as some of the main mechanism that can cause lung injury after chest trauma $(5,10)$.

PMN aggregation plays an important role in lung ischemiareperfusion injury. Neutrophils accumulate in the lung and release a variety of enzymes by degranulation; a large amount of oxygen free radicals are then generated in this process which induce endothelial cell damage and strong lipid peroxidation, finally leading to lung injury $(11,12)$. Our study found that, after treatment for $30 \mathrm{~min}, \mathrm{PMN}$ value differences in

Table VI. VEGF expression level in serum $(\mathrm{ng} / \mu \mathrm{l})$.

\begin{tabular}{|c|c|c|c|c|c|}
\hline Group & Cases & Before treatment & After treatment $1 / 2 \mathrm{~h}$ & T-value & P-value \\
\hline $\mathrm{RG}$ & 34 & $18.28 \pm 8.32$ & $4.58 \pm 2.19$ & 10.93 & 0.02 \\
\hline $\mathrm{CG}$ & 34 & $19.21 \pm 4.39$ & $18.38 \pm 3.39$ & 0.49 & 0.58 \\
\hline NG & 34 & $3.83 \pm 1.28$ & - & - & - \\
\hline T-value & - & 12.83 & 14.22 & - & - \\
\hline P-value & - & 0.01 & 0.008 & - & - \\
\hline
\end{tabular}

VEGF, vascular endothelial growth factor; RG, research group; CG, control group. 
peripheral blood of both research and control group are higher than before treatment. This result indicates that there was a certain degree of PMN aggregation in chest trauma patients' peripheral blood. However, after the mechanical ventilation treatment, the PMN value differences was decreased and lower than control group; differences were statistically significant $(\mathrm{P}<0.05)$. Therefore, mechanical ventilation treatment can decrease PMN aggregation and have a positive effect on improving this condition. Additionally, PVR levels of different times were significantly lower than the control group after treatment $(\mathrm{P}<0.05)$. $\mathrm{AaDO}_{2}$ values in control group after treatment for 1,3 and $6 \mathrm{~h}$ were all much lower than those in research group $(\mathrm{P}<0.05)$. MDA levels in research group was significantly lower than control group while the SOD and NO levels were much higher after treatment for $30 \mathrm{~min}$. This indicates that the anaerobic metabolism inside of chest trauma patients' body caused a significant increase of oxidative stress levels after ischemia-reperfusion injury, thereby reducing SOD but generating a large amount of ROS in the oxidative stress process, which can cause damage to the surrounding normal tissue. A timely treatment of mechanical ventilation can improve oxidative stress.

Moreover, Ang II levels in left atrial blood were much lower than in the control group $(\mathrm{P}<0.05)$ after treatment for $30 \mathrm{~min}$. Patients in this group experienced a shorter hospitalization time than control group; differences were statistically significant $(\mathrm{P}<0.05)$. Peripheral blood VEGF expression levels in RG was significantly lower than that in $\mathrm{CG}$; differences were statistically significant $(\mathrm{P}<0.05)$. This result showed that the VEGF levels also have a certain effect on the lung ischemia-reperfusion process.

It has been reported that there are a variety of control mechanisms of VEGF expression levels in the lung ischemia-reperfusion process $(13,14)$. Hypoxia and inflammation may lead to an increase in VEGF levels. Hypoxia-induced VEGF was mediated by hypoxia-inducible factor (HIF-1 $\alpha$ ) and combined with the core sequence of hypoxia response element in gene promoter so that it can be expressed (15). VEGF expression increases while the chest trauma is under hypoxia and ischemia conditions, but this more frequently occurs in alveolar epithelial cells. The alveolar cells damaged in the acute lung injury process can also contribute to the release of VEGF. Zhang et al found that acute inflammatory response can induce an increase in VEGF levels that are released at the early stage of acute lung injury (15-17). Alveolar macrophages and neutrophils are a potential source of VEGF expression, while VEGF can stimulate damaged alveolar macrophages and neutrophils migrate to lung tissue. Damaged alveolar macrophages and neutrophils can produce a large amount of VEGF is related to the effects of various inflammatory cytokines like TNF- $\alpha$, IL- 6 , IFN- $\alpha$, $\gamma$ (18). Therefore, after a lung injury, the number of alveolar macrophages and neutrophils are increased as are levels of pro-inflammatory cytokines, such as VEGF expression levels. However, an early treatment of mechanical ventilation can greatly improve the patient hypoxia-ischemia status to reduce the generation of VEGF, infiltration of inflammatory cells and release of inflammatory cytokines.

In conclusion, mechanical ventilation treatment for chest trauma patients can reduce the high VEGF expression levels in serum caused by ischemic-reperfusion and has good application prospects.

\section{References}

1. Liu L, Jiang Y and Steinle JJ: Compound 49b restores retinal thickness and reduces degenerate capillaries in the rat retina following ischemia/reperfusion. PLoS One 11: e0159532, 2016.

2. Jeong EK, Jang HJ, Kim SS, Lee SY, Oh MY, Kim HJ, Eom DW, Ham JY and Han DJ: Protective effect of polydeoxyribonucleotide against renal ischemia-reperfusion injury in mice. Transplant Proc 48: 1251-1257, 2016.

3. Tong F, Tang X,Li X, Xia W and Liu D: The effect of insulin-loaded linear poly(ethylene glycol)-brush-like poly(1-lysine) block copolymer on renal ischemia/reperfusion-induced lung injury through downregulating hypoxia-inducible factor. Int J Nanomed 11: 1717-1730, 2016

4. Li GH, Luo B, Lv YX, Zheng F, Wang L, Wei MX, Li XY, Zhang L, Wang JN, Chen SY, et al: Dual effects of VEGF-B on activating cardiomyocytes and cardiac stem cells to protect the heart against short- and long-term ischemia-reperfusion injury. J Transl Med 14: 116, 2016.

5. Allen BS, Ko Y, Buckberg GD and Tan Z: Studies of isolated global brain ischaemia: III. Influence of pulsatile flow during cerebral perfusion and its link to consistent full neurological recovery with controlled reperfusion following $30 \mathrm{~min}$ of global brain ischaemia. Eur J Cardiothorac Surg 41: 1155-1163, 2012.

6. Jiang Y, Li L, Ma J, Zhang L, Niu F, Feng T and Li C: Auricular vagus nerve stimulation promotes functional recovery and enhances the post-ischemic angiogenic response in an ischemia/reperfusion rat model. Neurochem Int 97: 73-82, 2016.

7. Hao P: Monitoring of renal ischemia reperfusion injury in rabbits by ultrasonic contrast and its relationship with expression of VEGF in renal tissue. Asian Pac J Trop Med 9: 188-192, 2016.

8. Lan CC, Peng CK, Tang SE, Wu SY, Huang KL and Wu CP: Anti-vascular endothelial growth factor antibody suppresses ERK and NF- $\kappa B$ activation in ischemia-reperfusion lung injury. PLoS One 11: e0159922, 2016.

9. Liu Z, Liu X, Huang Y and Zhao J: Intraoperative mechanical ventilation strategies in patients undergoing one-lung ventilation: a meta-analysis. Springerplus 5: 1251, 2016.

10. Deng C, Zhai Z, Wu D, Lin Q, Yang Y, Yang M, Ding H, Cao X, Zhang Q and Wang C: Inflammatory response and pneumocyte apoptosis during lung ischemia-reperfusion injury in an experimental pulmonary thromboembolism model. J Thromb Thrombolysis 40: 42-53, 2015.

11. Weissmann N, Sydykov A, Kalwa H, Storch U, Fuchs B, Mederos y Schnitzler M, Brandes RP, Grimminger F, Meissner M, Freichel M, et al: Activation of TRPC6 channels is essential for lung ischaemia-reperfusion induced oedema in mice. Nat Commun 3: 649, 2012

12. Lancellotti P, Tribouilloy C, Hagendorff A, Popescu BA, Edvardsen T, Pierard LA, Badano L and Zamorano JL; Scientific Document Committee of the European Association of Cardiovascular Imaging: Recommendations for the echocardiographic assessment of native valvular regurgitation: an executive summary from the European Association of Cardiovascular Imaging. Eur Heart J Cardiovasc Imaging 14: 611-644, 2013.

13. Marti HH and Risau W: Systemic hypoxia changes the organ-specific distribution of vascular endothelial growth factor and its receptors. Proc Natl Acad Sci USA 95: 15809-15814, 1998.

14. Lee JW, Bae SH, Jeong JW, Kim SH and Kim KW: Hypoxia-inducible factor (HIF-1)alpha: its protein stability and biological functions. Exp Mol Med 36: 1-12, 2004.

15. Zhang XD, Hai CX, Cai FL, Liang X, Liu R, Chen HL, Qin XJ and Feng AJ: Time course for expression of VEGF and its receptor and regulator levels of contraction and relaxation in increased vascular permeability of lung induced by phosgene. Inhal Toxicol 20: 805-812, 2008.

16. Thickett DR, Armstrong L, Christie SJ and Millar AB: Vascular endothelial growth factor may contribute to increased vascular permeability in acute respiratory distress syndrome. Am J Respir Crit Care Med 164: 1601-1605, 2001.

17. Webb NJ, Myers CR, Watson CJ, Bottomley MJ and Brenchley PE: Activated human neutrophils express vascular endothelial growth factor (VEGF). Cytokine 10: 254-257, 1998.

18. Cohen T, Nahari D, Cerem LW, Neufeld G and Levi BZ: Interleukin 6 induces the expression of vascular endothelial growth factor. J Biol Chem 271: 736-741, 1996. 